\title{
Correlation of histone methyl marks with circulating nucleosomes in blood plasma of cancer patients
}

\author{
UGUR GEZER $^{1}$, UFUK MERT ${ }^{1}$, EMRE ÖZGÜR $^{1}$, EBRU E. YÖRÜKER $^{1}$, \\ STEFAN HOLDENRIEDER $^{2}$ and NEJAT DALAY ${ }^{1}$ \\ ${ }^{1}$ Department of Basic Oncology, Oncology Institute, Istanbul University, Capa 34390, Istanbul, Turkey; \\ ${ }^{2}$ Institute of Clinical Chemistry and Pharmacology, University of Bonn, D-53127 Bonn, Germany
}

Received December 7, 2011; Accepted February 6, 2012

DOI: $10.3892 / \mathrm{ol} .2012 .600$

\begin{abstract}
Circulating DNA is present in plasma/serum, mainly complexed with histones as nucleosomes. The detection of circulating nucleosomes (cNUCs) in the peripheral blood may be a diagnostic modality for cancer-associated changes of modified histone tails in blood circulation. In the present study, we investigated the correlation between the trimethylation of H3 lysine 9 (H3K9me3) and H4 lysine 20 (H4K20me3), which are hallmarks of pericentric heterochromatin, and cNUCs in healthy subjects and patients with colorectal cancer (CRC) and multiple myeloma (MM). The plasma concentration of cNUCs was measured using the Cell-Death Detection ELISA kit. Histone methylation marks were detected using chromatin immunoprecipitation (ChIP), followed by quantitative PCR with pericentric satellite 2 as the target sequence. The results showed a high variation in the concentrations of cNUCs, with healthy subjects exhibiting the lowest levels (median 0.194), the CRC patients intermediate (median 0.25) and the MM patients the highest levels (median 0.648). However, the differences between the groups did not reach statistical significance ( $>00.05)$. Analysis using the Pearson's correlation test revealed a significant positive correlation between the concentration of cNUCs and H3K9me3 and H4K20me3 in the whole study group $(\mathrm{N}=57, \mathrm{p}<0.001$ for both histone marks). A study of the correlation between cNUCs and histone marks in the individual study groups demonstrated the correlation between cNUCs and H3K9me3 in CRC patients to be weak $(\mathrm{p}=0.046)$, indicating that circulating $\mathrm{H} 3 \mathrm{~K} 9 \mathrm{me} 3$ may be modified in $\mathrm{CRC}$ patients. The histone marks were normalized using the values of cNUCs. In agreement with the weak correlation between cNUCs and $\mathrm{H} 3 \mathrm{~K} 9 \mathrm{me} 3$ in CRC patients, H3K9me3 levels (median 0.047) were lowest in this group compared with the other two groups (0.06 in healthy subjects, 0.2 in MM patients,
\end{abstract}

Correspondence to: Dr Ugur Gezer, Department of Basic Oncology, Oncology Institute, Istanbul University, Capa 34390, Istanbul, Turkey

E-mail: ugurd@istanbul.edu.tr

Key words: cancer, blood plasma, circulating nucleosomes, histone methylation $\mathrm{p}=$ not significant). For H4K20me3, the median values were 0.022 in healthy subjects, 0.052 in CRC patients and 0.056 in $\mathrm{MM}$ patients. In conclusion, our findings indicate a marked correlation between cNUCs and histone methyl marks.

\section{Introduction}

Circulating DNA in serum or plasma is increasingly being recognized as a biomarker for cancer as it has been shown to bear the same genetic and epigenetic changes as the tumor tissues, indicating the possibility of creating minimally invasive diagnostic tests based on tumor-specific DNA markers $(1,2)$. Circulating DNA exists in plasma/serum as free unbound DNA, DNA complexed with histones as nucleosomes or DNA included in apoptotic bodies (3). Associating with proteins protects DNA against digestion by plasma and serum nucleases (4). The results of previous studies showed that patients with various types of tumors had DNA fragments mainly of 150-200, 400, 600 and 800 bp as mono- and oligonucleosomes (5-7).

Previous studies have investigated circulating nucleosomes (cNUCs) for their potential as diagnostic and prognostic biomarkers or usefulness in therapy monitoring (for review see ref. 3). The results of these studies have revealed that, although cancer patients have a generally higher level of cNUCs compared to healthy individuals, its diagnostic value is limited as various benign diseases were also often associated with an elevated serum level of nucleosomes. The prognostic value of pretherapeutic nucleosome concentrations has been demonstrated in univariate analyses $(8,9)$. cNUCs have been shown to be valuable for monitoring for the early estimation of efficacy of cytotoxic cancer therapy (3).

Another potential use of cNUCs involves its utility as a diagnostic modality in disease-associated quantitative changes of modified histone tails in blood circulation. In a previous study, we showed that methylated histone marks may be detected on cNUCs (10). In a subsequent study, we focused on two methyl marks, the trimethylation of $\mathrm{H} 3$ lysine 9 (H3K9me3) and H4 lysine 20 (H4K20me3), which are hallmarks of pericentric heterochromatin. Of these methyl marks, H4K20me3 was previously reported to be reduced in certain primary tumors and tumor cell lines (11). Our previous results have provided evidence that $\mathrm{H} 3 \mathrm{~K} 9 \mathrm{me} 3$ may be reduced in the 
circulating plasma of patients with colorectal cancer (CRC) when compared with healthy subjects or patients with multiple myeloma (MM) (12). In the present study, our aim was to analyze the correlation between cNUCs and two histone methyl marks.

\section{Materials and methods}

Study population. The study group comprised patients with histologically confirmed CRC ( $=25)$, MM $(\mathrm{N}=17)$ and healthy volunteers $(\mathrm{N}=15)$. Blood samples were obtained from CRC patients at surgery and from patients with MM prior to chemotherapy. The blood plasma was immediately separated from the cells using Ficoll-gradient centrifugation and stored in aliquots at $-80^{\circ} \mathrm{C}$. Patient characteristics with regard to age and gender are shown in Table I and the clinical characteristics of the CRC patients are shown in Table II. The study was approved by the Institutional Review Board of the Institute of Oncology of Istanbul University.

Quantitation of cNUCs. The concentrations of cNUCs in blood plasma were determined using the Cell-Death Detection ELISA kit (Roche Diagnostics, Mannheim, Germany), as previously reported (10). Briefly, we applied $20 \mu \mathrm{l}$ of plasma twice and the mean signal values, measured in optical density (OD), were considered to be the relative plasma concentrations.

Chromatin immunoprecipitation (ChIP) from blood plasma. The ChIP assay was performed as previously reported by our laboratory (12). Briefly, agarose beads were blocked with BSA and, following washing, the beads were pre-incubated with antibodies against the $\mathrm{H} 3 \mathrm{~K} 9 \mathrm{me} 3$ and H4K20me3 (Millipore, Temecula, CA, USA) for $4 \mathrm{~h}$ at $4^{\circ} \mathrm{C}$. Subsequently, $200 \mu \mathrm{l}$ of plasma was diluted into $800 \mu \mathrm{l}$ of the ChIP dilution buffer and was then added to the pelleted agarose beads that were pre-incubated with antibodies. Following overnight incubation at $4^{\circ} \mathrm{C}$, the beads were washed with low salt, high salt, $\mathrm{LiCl}$ and Tris/EDTA buffers. Finally, the chromatin was eluted by incubating the beads at $65^{\circ} \mathrm{C}$ and proteins were removed by treatment with proteinase K. ChIP DNA was then purified using an appropriate purification kit and stored at $-20^{\circ} \mathrm{C}$.

Quantitative real-time PCR ( $P P C R)$. H3K9me3- or H4K20me3-related ChIP plasma DNA was amplified using qPCR with satellite 2 as the target sequence, as previously reported (12). The PCR amplifications were performed twice and the mean values were calculated. The comparative $\Delta \mathrm{Ct}$ method was used for quantitation. A linear standard curve was generated through serial dilutions of human genomic DNA with a linear amplification (correlation coefficient $=0.99$ ). These dilution series were co-amplified in each PCR session and the relative concentration of H3K9me3- or H4K20me3-related nucleosomal DNA from a given sample was derived from the cross-over threshold $(\mathrm{Ct})$ values using this dilution standard.

Statistical analysis. The Pearson's correlation test was used to evaluate the correlation between cNUCs and histone methyl marks. The differences between the respective nucleosomes or histone methyl marks of the groups were compared in a
Table I. Characteristics of the study population.

\begin{tabular}{lrrrrrrr}
\hline & & \multicolumn{2}{c}{ Gender } & & \multicolumn{2}{c}{ Age (years) } \\
\cline { 3 - 4 } \cline { 7 - 8 } & N & Male & Female & & Mean & Range \\
\hline Controls & 15 & 8 & 7 & & 41 & $24-65$ \\
Colorectal cancer & 25 & 15 & 10 & & 64 & $48-83$ \\
Multiple myeloma & 17 & 6 & 11 & & 62 & $46-82$ \\
& & & & & & &
\end{tabular}

Table II. Clinical characteristics of colorectal cancer and multiple myeloma patients.

$\begin{array}{lr}\text { Colorectal cancer } & \\ \text { Tumor localization } & 8 \\ \text { Colon } & 17 \\ \text { Rectum } & \\ \text { Stage (UICC) } & 7 \\ \text { I-II } & 18 \\ \text { III-IV }\end{array}$

Multiple myeloma

Stage (Durie-Salmon)

I-II

III

10

UICC, International Union Against Cancer.

univariate analysis using the Mann-Whitney $\mathrm{U}$ test. $\mathrm{P}<0.05$ was considered to indicate a statistically significant result.

\section{Results and Discussion}

We measured the concentration of cNUCs in the plasma of individual study participants (Fig. 1A) and detected a high variation, with a range of $0.098-2.5$. The healthy controls had the lowest relative levels of cNUCs (median, 0.194), the CRC patients intermediate (median, 0.25) and the MM patients the highest levels (median, 0.648). However, the difference between the healthy individuals and the CRC patients did not reach statistical significance $(\mathrm{p}=0.5)$, while for the difference between the healthy group and MM patients there was a trend towards significance $(\mathrm{p}=0.075)$. The finding that $\mathrm{CRC}$ and $\mathrm{MM}$ patients have higher levels of cNUCs than healthy subjects is consistent with previous reports $(3,13)$.

ChIP assays were performed to precipitate H3K9me3 and H4K20me3-related cNUCs and qPCR to quantitate satellite 2. The number of methyl marks should be normalized since the levels of cNUCs vary highly between individual samples. The method of data normalization has a major impact on the quality of ChIP analyses (14). In ChIP assays within the context of cell or tissues, 'input' controls (e.g., total chromatin) or pan antibodies for histones (e.g., histone H3 or H4) are used to normalize the target protein or modification (15). This 
A

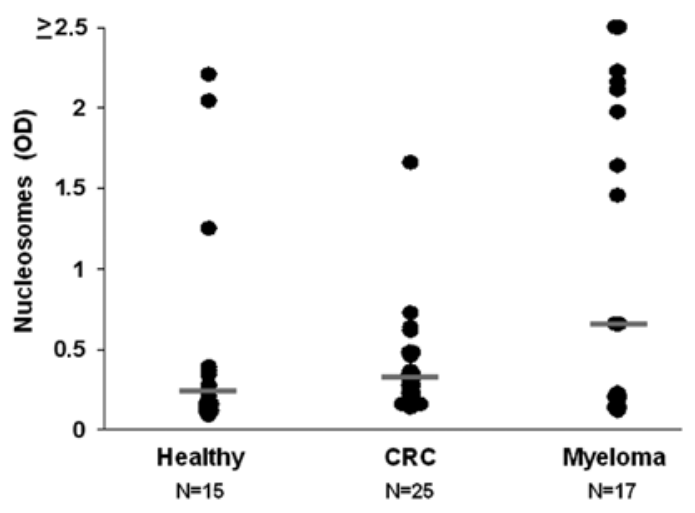

B

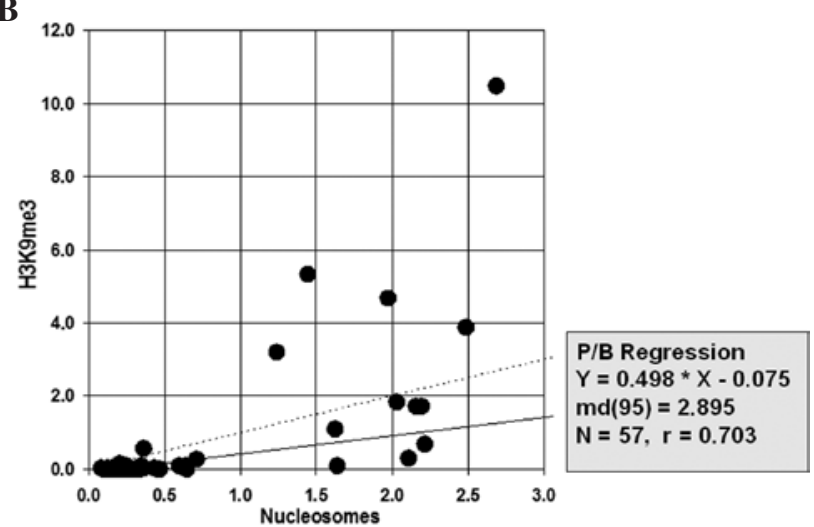

C

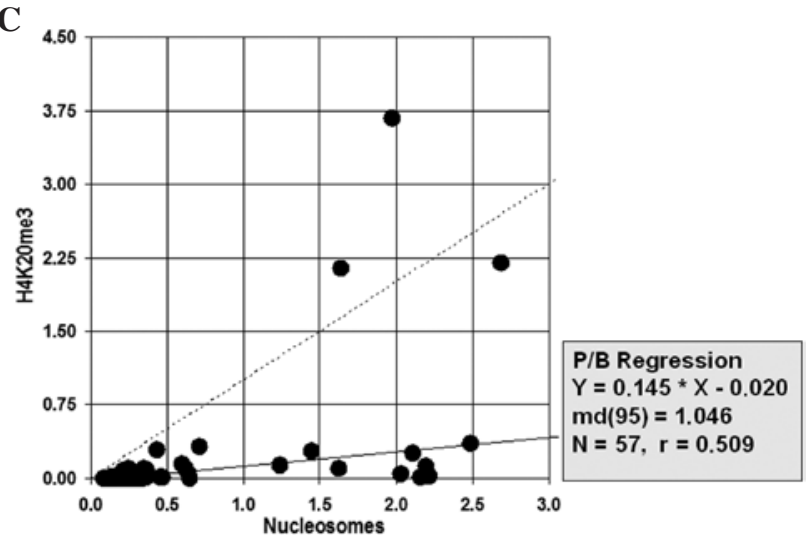

Figure 1. Levels of circulating nucleosomes and their correlation with histone methyl marks are shown. (A) Levels of nucleosomes were determined using the Cell-Death Detection ELISA kit using $20 \mu \mathrm{l}$ of plasma twice. The mean signal values, measured in $\mathrm{OD}$, were considered to be the relative concentrations in plasma. The correlation between nucleosomes and (B) H3K9me3 and (C) H4K20me3 was calculated using Pearson's test in the whole study population $(\mathrm{N}=57)$. OD, optical density.

process, however, is not appropriate for blood fluids (serum, plasma) as not all the circulating DNA is associated with histones in the form of nucleosomes (3). Thus, using the total plasma DNA for normalization may lead to inaccurate results. Similarly, the pool of cNUCs is likely to include modified and unmodified forms of the histone residue of interest, hampering the normalization of the target modification. Therefore, we considered total nucleosomes for normalizing the circulating levels of methyl marks.
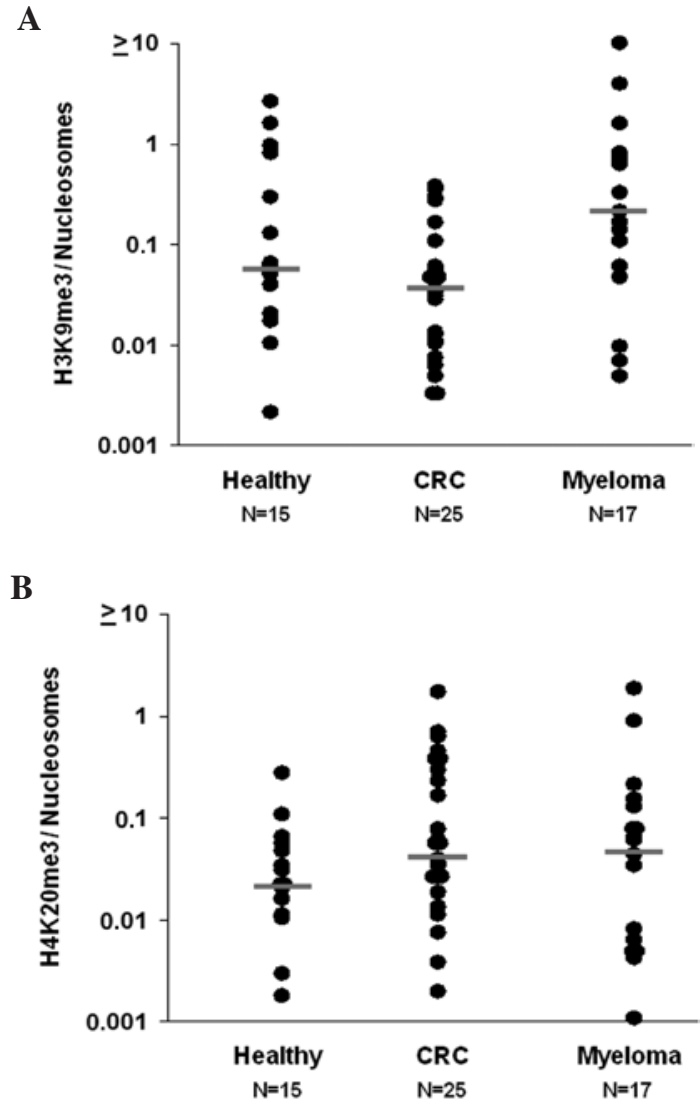

Figure 2. Levels of histone methyl marks. (A) H3K9me3 and (B) H4K20me3 were determined by ChIP assays using specific antibodies and qPCR results were normalized using the levels of nucleosomes. The y-axis is scaled logarithmically. ChIP, chromatin immunoprecipitation; qPCR, quantitative real-time PCR.

To ascertain whether cNUCs are suitable for that purpose we first determined whether there was a correlation between cNUCs and histone marks. Figs. 1B and C show the correlation curves for the whole study group $(\mathrm{N}=57)$. Analysis with the Pearson's test revealed a significant positive correlation between cNUCs and H3K9me3 or H4K20me3 ( $<<0.001$ for both histone marks). This correlation indicates that histone marks may be normalized using the values of cNUCs. However, when we studied the correlation between cNUCs and histone marks in individual study groups, the correlation between cNUCs and $\mathrm{H} 3 \mathrm{~K} 9 \mathrm{me} 3$ in the CRC patients was found to deviate from that of the whole study group and was at the limit of significance $(\mathrm{p}=0.046)$. This reveals that the amount of $\mathrm{H} 3 \mathrm{~K} 9 \mathrm{me} 3$ in circulation may be modified in CRC patients.

Figs. 2A and B show H3K9me3 and H4K20me3 levels normalized by cNUCs, respectively. In agreement with the weak correlation between cNUCs and H3K9me3 in CRC patients, H3K9me3 levels were lowest in this group (0.047), whereas the values were 0.06 in healthy subjects and 0.2 in MM patients. The difference between the healthy group and MM patients was, however, not significant $(\mathrm{p}=0.38)$. The distribution of $\mathrm{H} 3 \mathrm{~K} 9 \mathrm{me} 3$ in the CRC patients was different from that of the MM patients $(\mathrm{p}=0.044)$. For H4K20me3, the median values were 0.022 in the healthy subjects, 0.052 in the CRC patients ( $\mathrm{p}=0.07)$ and 0.056 in the MM patients $(\mathrm{p}=0.36)$. 
In conclusion, this pilot study is the first to assess the correlation between cNUCs and histone methyl marks. Our findings indicate that there is a marked positive correlation between these parameters. In addition, the normalizing circulating levels of $\mathrm{H} 3 \mathrm{~K} 9 \mathrm{me} 3$ and $\mathrm{H} 4 \mathrm{~K} 20 \mathrm{me} 3$ confirm our previous finding that $\mathrm{H} 3 \mathrm{~K} 9 \mathrm{me} 3$ may be reduced in $\mathrm{CRC}$ patients (12). Studies with larger sample sizes are required to confirm and validate the potential of these findings in CRC.

\section{Acknowledgements}

This study was supported by the Scientific Research Projects Coordination Unit of Istanbul University (project number 17758).

\section{References}

1. Beck J, Urnovitz HB, Mitchell WM and Schütz E: Next generation sequencing of serum circulating nucleic acids from patients with invasive ductal breast cancer reveals differences to healthy and nonmalignant controls. Mol Cancer Res 8: 335-342, 2010.

2. Vlassov VV, Laktionov PP and Rykova EY: Circulating nucleic acids as a potential source for cancer biomarkers. Curr Mol Med 10: 142-165, 2010.

3. Holdenrieder S and Stieber P: Clinical use of circulating nucleosomes. Crit Rev Clin Lab Sci 46: 1-24, 2009.

4. Ng EK, Tsui NB, Lam NY, Chiu RW, Yu SC, Wong SC, Lo ES, Rainer TH, Johnson PJ and Lo YM: Presence of filterable and nonfilterable mRNA in the plasma of cancer patients and healthy individuals. Clin Chem 48: 1212-1217, 2002.

5. Giacona MB, Ruben GC, Iczkowski KA, Roos TB, Porter DM and Sorenson GD: Cell-free DNA in human blood plasma: length measurements in patients with pancreatic cancer and healthy controls. Pancreas 17: 89-97, 1998.
6. Jahr S, Hentze H, Englisch S, Hardt D, Fackelmayer FO, Hesch RD and Knippers R: DNA fragments in the blood plasma of cancer patients: quantitations and evidence for their origin from apoptotic and necrotic cells. Cancer Res 61: 1659-1665, 2001.

7. Deligezer U, Yaman F, Erten N and Dalay N: Frequent copresence of methylated DNA and fragmented nucleosomal DNA in plasma of lymphoma patients. Clin Chim Acta 335: 89-94, 2003.

8. Holdenrieder S, Stieber P, von Pawel J, Raith H, Nagel D, Feldmann K and Seidel D: Circulating nucleosomes predict the response to chemotherapy in patients with advanced non-small cell lung cancer. Clin Cancer Res 10: 5981-5987, 2004.

9. Kremer A, Holdenrieder S, Stieber P, Wilkowski R, Nagel D and Seidel D: Nucleosomes in colorectal cancer patients during radiochemotherapy. Tumor Biol 27: 235-242, 2006.

10. Deligezer U, Akisik EE, Erten N and Dalay N: Sequence-specific histone methylation is detectable on circulating nucleosomes in plasma. Clin Chem 54: 1125-1131, 2008.

11. Fraga MF, Ballestar E, Villar-Garea A, Boix-Chornet M, Espada J, Schotta G, Bonaldi T, Haydon C, Ropero S, Petrie K, et al: Loss of acetylation at Lys16 and trimethylation at Lys20 of histone $\mathrm{H} 4$ is a common hallmark of human cancer. Nat Genet 37: 391-400, 2005.

12. Deligezer U, Akisik EZ, Akisik EE, Kovancilar M, Bugra D, Erten $\mathrm{N}$, Holdenrieder S and Dalay N: H3K9me3/H4K20me3 ratio in circulating nucleosomes as potential biomarker for colorectal cancer. In: Circulating Nucleic Acids in Plasma and Serum. Gahan PB (ed). Springer Books, Amsterdam, pp97-103, 2011.

13. Holdenrieder S, von Pawel J, Dankelmann E, Duell T, Faderl B, Markus A, Siakavara M, Wagner H, Feldmann K, Hoffmann H, et al: Nucleosomes and CYFRA 21-1 indicate tumor response after one cycle of chemotherapy in recurrent non-small cell lung cancer. Lung Cancer 63: 128-135, 2009.

14. Haring M, Offermann S, Danker T, Horst I, Peterhansel C and Stam M: Chromatin immunoprecipitation: optimization, quantitative analysis and data normalization. Plant Methods 3: 11, 2007.

15. Jayani RS, Ramanujam PL and Galande S: Studying histone modifications and their genomic functions by employing chromatin immunoprecipitation and immunoblotting. Methods Cell Biol 98: 35-56, 2010. 